\title{
Function Series, Catalan Numbers, and Random Walks on Trees
}

Ibtesam Bajunaid, Joel M. Cohen, Flavia Colonna, David Singman

1. INTRODUCTION. The delight of finding unexpected connections is one of the rewards of studying mathematics. In this paper we present connections that link the following seven superficially unrelated entities:

(A) A function of the sort that calculus textbooks often use to show that a continuous function need not have a derivative at each point (see Figure 1):

$$
f(p)= \begin{cases}1 & \text { if } p \in[0,1 / 2] \\ \frac{1-p}{p} & \text { if } p \in[1 / 2,1]\end{cases}
$$

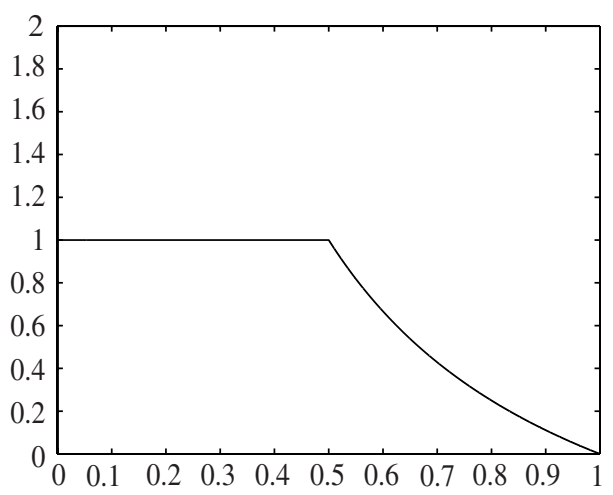

Figure 1.

(B) A series

$$
\sum_{n=0}^{\infty} a_{n}[p(1-p)]^{n}
$$

that converges on the interval $[0,1]$ to a continuous function of $p$ that fails to be differentiable at $p=1 / 2$.

(C) An infinite sequence $1,1,2,5,14,42,132, \ldots$ that can be obtained from a triangle with a Pascal-like addition rule. The numbers in the sequence are the nonzero entries in the first column:

$\begin{array}{ccccccc}1 & & & & & & \\ 0 & 1 & & & & & \\ 1 & 0 & 1 & & & & \\ 0 & 2 & 0 & 1 & & & \\ 2 & 0 & 3 & 0 & 1 & & \\ 0 & 5 & 0 & 4 & 0 & 1 & \\ 5 & 0 & 9 & 0 & 5 & 0 & 1 \\ \ldots & \ldots & \ldots & \ldots & \ldots & \ldots & \ldots\end{array}$


(D) A sequence $a_{n}(n=0,1, \ldots)$ that can be described by means of the recursion formula

$$
a_{n+1}=\sum_{k=0}^{n} a_{n-k} a_{k} \quad(n \geq 0)
$$

and the initial condition $a_{0}=1$.

(E) The equation $|z(1-z)|=1 / 4$, or equivalently, $\left(x^{2}+y^{2}\right)\left[(1-x)^{2}+y^{2}\right]=$ $1 / 16$, whose graph looks like a figure-eight (see Figure 2 ):

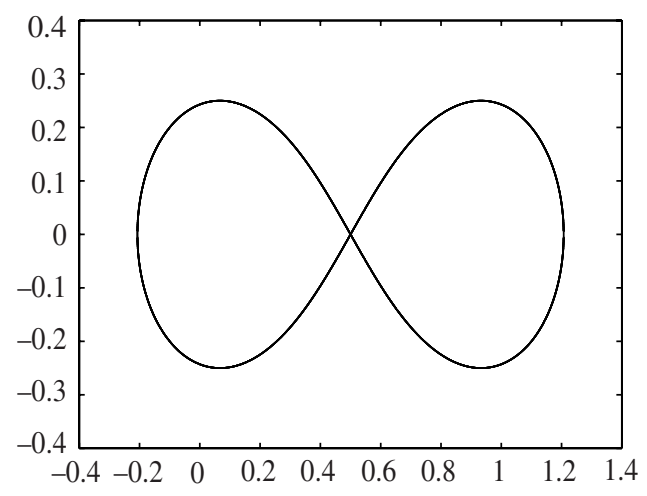

Figure 2.

(F) The classical probability problem of the "drunkard's walk," which asks for the probability that a path on the nonnegative integers starting at some point ends up at 0 .

(G) A functional equation of the form $f(p)=(1-p)+p f(p)^{2}$.

The numbers of the infinite sequence in (C), known as the Catalan numbers, have many combinatorial interpretations [7]. They satisfy the recursion relation (D). In section 3 we show that the Catalan numbers are the coefficients of the series in $(\mathrm{B})$, whose sum $h(p)$ coincides with $f(p) /(1-p)$ on the interval $[0,1]$, where $f$ is the function described in (A). Thus, the series gives a single formula for the function defined piecewise in (1). If we regard the series as being defined over $\mathbb{C}$ and determine its set of convergence $K$, we are led to the equation in (E), whose graph bounds $K$. The drunkard's walk $(\mathrm{F})$ is the source of both $f$ and its series representation. This function $f$ satisfies the functional relation $(\mathrm{G})$.

In section 2 we introduce trees and random walks on trees. In section 3 we do all the calculations that make precise the connections outlined in the previous paragraph. In sections 4 and 5 we find an infinite sequence of entities analogous to (A), (B), (C), (D), (E), (F), and (G) with exactly the same types of connections.

In particular, we introduce the "cautious drunkard's walk," which can be viewed either as a random walk on the nonnegative integers with jumps or as a random walk without jumps on a certain directed graph. We describe this directed graph, which yields the probabilistic interpretation of the sequence introduced in section 4 .

Furthermore, we describe a relation among the sequences of numbers given by (C) and its generalizations. Finally, in section 6 we present the problem that led us to this whole study. 
2. RANDOM WALKS ON TREES. We first recall some basic terminology. A tree $T$ is a connected graph with no loops such that each vertex is an endpoint of only finitely many edges. Two distinct vertices $v$ and $w$ of $T$ are called neighbors if there is an edge connecting them, in which case we signify this relationship by writing $v \sim w$.

A path in $T$ is a finite or infinite sequence of vertices $\left[v_{0}, v_{1}, \ldots\right]$ such that $v_{k} \sim v_{k+1}$ for all $k$. A path is called a geodesic if $v_{k-1} \neq v_{k+1}$ holds for $k=1,2, \ldots$ An infinite geodesic is called a ray emanating from its initial point $v_{0}$. If $u$ and $v$ are vertices of $T$, we denote by $[u, v]$ the (necessarily unique) geodesic $\left[w_{0}, w_{1}, \ldots, w_{n}\right]$ such that $w_{0}=u$ and $w_{n}=v$.

It is frequently convenient to single out a specific vertex $e$ of a tree and declare it to be a root of the tree. There is a natural distance function $d$ defined on the set of vertices of a tree $T$ : if $u$ and $v$ are vertices, $d(u, v)$ is the number of edges in the geodesic from $u$ to $v$. The length $|v|$ of a vertex $v$ of a tree with root $e$ is its distance $d(v, e)$ from $e$. The predecessor $v^{-}$of a vertex $v$ different from $e$ is the next to last vertex of the geodesic path from $e$ to $v$. A descendant of a vertex $v$ is a vertex $u$ such that $v$ is on the path from $e$ to $u$.

Let $p$ be a nearest-neighbor transition probability on $T$, that is, $p(v, u) \geq 0$ if $v$ and $u$ are neighboring vertices, $p(v, u)=0$ if $v$ and $u$ are not neighbors, and, for all vertices $v, \sum_{u \sim v} p(v, u)=1$. If $\gamma=\left[v_{0}, \ldots, v_{n}\right]$ is a finite path, we define the probability of $\gamma$ relative to $p$ by

$$
p(\gamma)=\prod_{j=1}^{n} p\left(v_{j-1}, v_{j}\right)
$$

with $p\left(\left[v_{0}\right]\right)=1$. This defines a random walk on $T$ as follows.

Let $W$ be the set of all infinite paths. For each finite path $\gamma$ let the cylinder $W(\gamma)$ be the set of all paths in $W$ whose initial segment is $\gamma$. Fix a vertex $v$. Let $\mathcal{F}_{v}$ be the $\sigma$-algebra generated by the cylinders $W(\gamma)$ for which $\gamma$ is a path beginning at $v\left(\mathcal{F}_{v}\right.$ is the smallest collection of subsets of $W$ containing all such cylinders that is closed under countable unions and complements). Then there exists a unique probability measure $P_{v}$ on $\mathcal{F}_{v}$ such that $P_{v}(W(\gamma))=p(\gamma)$ for each such $\gamma$ [2, sec. 3.1]. The random walk on $T$ associated with $p$ consists of the set of all paths in $W$ together with each $P_{v}$. We can calculate the probability that a path satisfies a given property by evaluating some $P_{v}$ on the set of all paths with this property, provided that such a set is in $\mathcal{F}_{v}$.

For vertices $v$ and $w$ of $T, \Gamma_{v, w}$ denotes the set of finite paths of positive length from $v$ to $w$ that visit $w$ after the first step only once (i.e., $\Gamma_{v, w}$ is the set of all paths $\left[v=v_{0}, \ldots, v_{n}=w\right]$ with $n \geq 1$ and $v_{j} \neq w$ when $0<j<n$ ). Let $W_{v, w}$ be the set of all infinite paths beginning at $v$ and containing $w$. Observe that $W_{v, w}$ is the disjoint union of all the cylinders $W(\gamma)$, where $\gamma$ lies in $\Gamma_{v, w}$. Thus $W_{v, w}$ belongs to $\mathcal{F}_{v}$, so $F(v, w)=P_{v}\left(W_{v, w}\right)$ is defined. This is the probability that a random walk beginning at $v$ visits $w$ in positive time. Notice that

$$
F(v, w)=\sum_{\gamma \in \Gamma_{v, w}} p(\gamma)
$$

A random walk on $T$ is recurrent if $F(v, w)=1$ for all vertices $v$ and $w$. A nonrecurrent random walk is transient. Transient random walks have the property that with probability one an infinite path will visit any particular vertex only finitely many times.

There is a simple test for transience of a random walk on an arbitrary tree $T$ rooted at $e$ with a nearest-neighbor transition probability $p$. If $\rho=\left[e=w_{0}, w_{1}, \ldots\right]$ is a ray 
starting at $e$, let

$$
\epsilon_{n}(\rho)=\left\{\begin{array}{cl}
\frac{p\left(w_{n}, w_{n-1}\right)}{1-p\left(w_{n}, w_{n-1}\right)} & \text { if } n \geq 1 \\
1 & \text { if } n=0
\end{array}\right.
$$

and let

$$
H^{*}(\rho)=\sum_{k=0}^{\infty} \epsilon_{0}(\rho) \epsilon_{1}(\rho) \cdots \epsilon_{k}(\rho) .
$$

In [1] (see Theorems 5.1(a) and 5.2), the authors established the following test for transience:

\section{Theorem 2.1.}

(a) If a random walk on a tree $T$ rooted at e is transient, then $H^{*}(\rho)<\infty$ for some ray $\rho$ emanating from $e$.

(b) If there exists a geodesic path $c=\left[e=w_{0}, \ldots, w_{n}\right]$ such that $H^{*}(\rho)<\infty$ for all rays $\rho$ beginning with $c$, then the random walk on $T$ is transient.

In particular, we have:

Proposition 2.1. Let $T$ be a tree rooted at e, and let $r$ belong to $(0,1 / 2)$. Assume that there exists a vertex $v_{0}$ of $T$ such that $p\left(u, u^{-}\right) \leq r$ for each descendant $u$ of $v_{0}$. Then the random walk on $T$ corresponding to $p$ is transient.

We show in section 6 that Theorem 2.1 is sharp (i.e., $H^{*}$ by itself does not always determine transience). The function $F$ defined in (2) exhibits the following easily verified, but very useful properties (see [2, secs. 2.3 and 2.4]):

Proposition 2.2. Let $v$ and $w$ be distinct vertices of a tree $T$, and let $\left[v_{0}, \ldots, v_{n}\right]$ be the geodesic path with $v_{0}=v$ to $v_{n}=w$. Then the following statements are true about the function $F$ given by (2):

(a) $F(v, w)=\prod_{k=0}^{n-1} F\left(v_{k}, v_{k+1}\right)$;

(b) $F(v, v)=\sum_{u \sim v} p(v, u) F(u, v)$;

(c) If $v \sim w$, then $F(v, w)=p(v, w)+\sum_{u \sim v, u \neq w} p(v, u) F(u, w)$.

It turns out that the transience of a random walk is equivalent to the condition that $F(v, v)<1$ for all vertices $v$ :

Proposition 2.3. If $F\left(v_{0}, v_{0}\right)=1$ for some vertex $v_{0}$ of $T$, then the random walk on $T$ associated with $F$ is recurrent.

Proof. Let

$$
r(v)=\sum_{n=0}^{\infty} F(v, v)^{n} \leq \infty .
$$

Then $r(v)$ is the expected number of all visits to $v$ starting at $v$. Indeed if $F(v, v)=1$ this is obvious. If $F(v, v)<1$, since $F(v, v)^{n}$ is the probability that a path starting at 
$v$ returns at least $n$ times, the expected number of visits to $v$ is

$$
\sum_{n=1}^{\infty} n\left[F(v, v)^{n-1}-F(v, v)^{n}\right]=\sum_{n=0}^{\infty}(n+1) F(v, v)^{n}-\sum_{n=0}^{\infty} n F(v, v)^{n}=r(v) .
$$

Assume that $F\left(v_{0}, v_{0}\right)=1$, so that $r\left(v_{0}\right)=\infty$. Let $v$ be an arbitrary vertex, let $\gamma=$ $\left[w_{0}=v, w_{1}, \ldots, w_{n}=v_{0}\right]$ be a path from $v$ to $v_{0}$, and let $\gamma^{-1}=\left[w_{n}, w_{n-1}, \ldots, w_{0}\right]$ be the reverse of $\gamma$. Then the expected number of times that a path starting at $v$ passes through $v_{0}$ and ends at $v$ is $p(\gamma) r\left(v_{0}\right) p\left(\gamma^{-1}\right)=\infty$, so $r(v)=\infty$. This implies that $F(v, v)=1$. From Proposition 2.2(b) it follows that $F(u, v)=1$ if $u \sim v$. Invoking part (a) of the same result, we obtain $F(u, w)=1$ for all vertices $u$ and $w$ of $T$.

In contrast to Proposition 2.3, it is possible to have a transient random walk for which $F(v, w)=1$ for some $v \neq w$. The example in section 6 demonstrates this.

By a homogeneous tree of degree $d(d \geq 2)$ we mean a tree all of whose vertices have exactly $d$ neighbors. This concept is pertinent to the following two examples.

Example 2.1. Let $T$ be a homogeneous tree of degree $d(d \geq 3)$. We assign the isotropic nearest-neighbor transition probability: $p(u, v)=1 / d$ if $u$ and $v$ are neighbors. By Proposition 2.1, the random walk on $T$ corresponding to $p$ is transient.

Example 2.2. Now let $T_{3}$ be a homogeneous tree of degree three rooted at $e$, and define a nearest-neighbor transition probability as follows: if $v$ is a vertex other than $e$, let $p(e, v)=1 / 3$ if $|v|=1, p\left(v^{-}, v\right)=1 / 4$ if $|v| \geq 2$, and $p\left(v, v^{-}\right)=1 / 2$ if $|v| \geq 1$. For any ray $\rho$ emanating from $e, \epsilon_{n}(\rho)=1$ for each nonnegative integer $n$, so $H^{*}(\rho)=\infty$. Thus, by Theorem 2.1, the induced random walk on $T_{3}$ corresponding to $p$ is recurrent.

In sections 3 and 4 we shall study some nearest-neighbor random walks on $\mathbb{Z}_{+}^{0}=$ $\mathbb{Z}_{+} \cup\{0\}$, where $\mathbb{Z}_{+}=\{1,2, \ldots\}$. For such random walks, recurrence is completely determined by the value of $F(1,0)$ as the following proposition shows.

Proposition 2.4. A nearest-neighbor random walk on $\mathbb{Z}_{+}^{0}$ is recurrent if and only if $F(1,0)=1$.

Proof. First observe that if the random walk is recurrent, then by definition

$$
F(1,0)=1 .
$$

Conversely, assume that $F(1,0)=1$. Let $p=p(1,2)$, and note that $p(0,1)=1$ and $p(1,0)=1-p$. By Proposition 2.2(c) we see that

$$
F(1,0)=1-p+p F(2,0),
$$

so $F(2,0)=1$. But by Proposition $2.2(\mathrm{a}), F(2,0)=F(2,1) F(1,0)$, implying that $F(2,1)=1$. On the other hand, Proposition 2.2(b) gives

$$
F(1,1)=1-p+p F(2,1)=1 .
$$

According to Proposition 2.3, the random walk is recurrent.

3. THE DRUNKARD'S WALK ON $\mathbb{Z}_{+}^{0}$. In this section we study in detail the drunkard's walk (F) on $\mathbb{Z}_{+}^{0}$ and establish the connections between items (A) through $(\mathrm{G})$. The drunkard's walk with parameter $p(0<p<1)$ is a random walk on $\mathbb{Z}_{+}^{0}$ such that $p(n, n+1)=p$ and $p(n, n-1)=1-p$ when $n \geq 1$ and $p(0,1)=1$. We imagine 
that if the drunkard is at $n$, then he moves forward with probability $p$ and backward with probability $1-p$. We want to know the probability that starting at 1 he will sooner or later "fall in the lake" (i.e., touch the point 0 ). We consider $\mathbb{Z}_{+}^{0}$ as a tree with edges $[n, n+1]$ for $n \geq 0$. Thus we wish to calculate $F(1,0)$, the probability that a walk beginning at 1 touches 0 .

A path starting at 1 and visiting 0 for the first time is a loop in $\mathbb{Z}_{+}$that begins and ends at 1 and that is followed by one step from 1 to 0 . In order to calculate $F(1,0)$, we first consider the set of loops in $\mathbb{Z}_{+}$.

For $n=0,1,2, \ldots$ we use $A_{n}$ to signify the set of finite sequences

$$
c=\left[c_{0}, c_{1}, \ldots, c_{2 n}\right]
$$

in $\mathbb{Z}_{+}$such that $c_{0}=c_{2 n}=1$ and $\left|c_{k}-c_{k+1}\right|=1$ for $k=0,1, \ldots, 2 n-1$. These are the paths in $\mathbb{Z}_{+}$that begin and end at 1 (i.e., the loops in $\mathbb{Z}_{+}$based at 1 ). Let $a_{n}$ be the cardinality of $A_{n}$, and for $m$ in $\mathbb{Z}_{+}$let $\gamma_{n, m}$ be the number of paths in $\mathbb{Z}_{+}$of length $n$ starting at $m$ and ending at 1 . Then $a_{n}=\gamma_{2 n, 1}$. Obviously, $\gamma_{0,1}=1$ and

$$
\gamma_{n, m}=\gamma_{n-1, m-1}+\gamma_{n-1, m+1}
$$

When $n>0$ and $m>1$ this readily yields the entries in the one-sided Pascal triangle in $(\mathrm{C})$.

A path of length $2 n+1$ that enters into the calculation of $F(1,0)$ consists of $n$ steps to the right, each having probability $p$, and $n+1$ steps to the left, each having probability $1-p$. There are $a_{n}$ such paths, so

$$
F(1,0)=\sum_{n=0}^{\infty} a_{n} p^{n}(1-p)^{n+1}=(1-p) \sum_{n=0}^{\infty} a_{n}[p(1-p)]^{n},
$$

which is $1-p$ times the series in (B).

We use two different methods to express the coefficients $a_{n}$ in closed form. The first is purely combinatorial, the second algebraic.

A Dyck path of length $k$ is a finite sequence $\left(a, b_{0}\right),\left(a+1, b_{1}\right), \ldots,\left(a+k, b_{k}\right)$ in $\mathbb{Z} \times \mathbb{Z}$, where $b_{i+1}=b_{i} \pm 1$. With a path $c=\left[c_{0}, \ldots, c_{2 n}\right]$ in $A_{n}$ we associate the Dyck path

$$
(0,1)=\left(0, c_{0}\right),\left(1, c_{1}\right), \ldots,\left(2 n, c_{2 n}\right)=(2 n, 1)
$$

This establishes a one-to-one correspondence between $A_{n}$ and the set of Dyck paths from $(0,1)$ to $(2 n, 1)$ that remain in $\mathbb{Z}_{+}^{0} \times \mathbb{Z}_{+}$. The number of such Dyck paths is the total number of Dyck paths from $(0,1)$ to $(2 n, 1)$ in $\mathbb{Z} \times \mathbb{Z}$ less the number of these that touch the $x$-axis. But by a well-known reflection principle (see [3, p. 72]) a Dyck path from $(0,1)$ to $(2 n, 1)$ touching the $x$-axis corresponds to an arbitrary Dyck path from $(0,-1)$ to $(2 n, 1)$ : to see this, simply reflect across the $x$-axis that part of the latter path between $(0,-1)$ and the first point at which it touches the $x$-axis. The number of all Dyck paths from $(0,1)$ to $(2 n, 1)$ is the number of ways of moving up $n$ times and down $n$ times in $2 n$ moves, which is $\left(\begin{array}{c}2 n \\ n\end{array}\right)$. On the other hand, the number of Dyck paths from $(0,-1)$ to $(2 n, 1)$ is the number of ways of moving up $n+1$ times and down $n-1$ times in $2 n$ moves, which is $\left(\begin{array}{c}2 n \\ n-1\end{array}\right)$. Thus

$$
a_{n}=\left(\begin{array}{c}
2 n \\
n
\end{array}\right)-\left(\begin{array}{c}
2 n \\
n-1
\end{array}\right)=\left(\begin{array}{c}
2 n \\
n
\end{array}\right)-\frac{n}{n+1}\left(\begin{array}{c}
2 n \\
n
\end{array}\right)=\frac{1}{n+1}\left(\begin{array}{c}
2 n \\
n
\end{array}\right) \text {. }
$$


A second way of calculating $a_{n}$ is to find a recurrence relation satisfied by these numbers and then exploit it to derive the generating function for the sequence. To this end, we define a function $\sigma: A_{n} \rightarrow A_{n+1}$ as follows: for $c=\left[c_{0}, c_{1}, \ldots, c_{2 n}\right]$ in $A_{n}$, let

$$
\sigma(c)=\left[1, c_{0}+1, c_{1}+1, \ldots, c_{2 n}+1,1\right]
$$

Clearly, $\sigma$ is one-to-one, and its image is the set of paths in $A_{n+1}$ that revisit 1 only at the last step. Next we define the concatenation $c \cdot d$ of paths $c$ in $A_{n}$ and $d$ in $A_{m}$ to be the path in $A_{n+m}$ given by $c \cdot d=\left[c_{0}, c_{1}, \ldots, c_{2 n}, d_{1}, \ldots, d_{2 m}\right]$. Armed with this notation, we are in position to handle (D):

Theorem 3.1. The sets $A_{n}$ are described inductively as follows: $A_{0}=\{[1]\}$ and for each nonnegative integer $n A_{n+1}$ is the disjoint union of the sets of paths $\sigma\left(A_{k}\right) \cdot A_{n-k}$ for $k=0,1, \ldots, n$. In particular, $a_{0}=1$ and

$$
a_{n+1}=\sum_{k=0}^{n} a_{k} a_{n-k}
$$

for $n=0,1, \ldots$

Proof. Any $c$ in $A_{n+1}$ can be written as $\left[c_{0}, c_{1}, \ldots, c_{2 k+2}, c_{2 k+3}, \ldots, c_{2 n+2}\right]$, where $c_{2 k+2}$ is the first occurrence of 1 after $c_{0}$. This yields a unique representation of $c$ as a concatenation $c^{\prime} \cdot c^{\prime \prime}$, where

$$
c^{\prime}=\left[c_{0}, \ldots, c_{2 k+2}\right]=\sigma\left(\left[c_{1}-1, \ldots, c_{2 k+1}-1\right]\right)
$$

belongs to $\sigma\left(A_{k}\right)$ and $c^{\prime \prime}=\left[c_{2 k+2}, c_{2 k+3}, \ldots, c_{2 n+2}\right]$ to $A_{n-k}$. Conversely, any elements of $\sigma\left(A_{k}\right)$ and $A_{n-k}$ can plainly be concatenated to yield an element in $A_{n+1}$.

Theorem 3.2. The power series $\sum_{n=0}^{\infty} a_{n} z^{n}$ converges when $|z| \leq 1 / 4$, and its sum $g(z)$ is given by

$$
g(z)= \begin{cases}\frac{1-\sqrt{1-4 z}}{2 z} & \text { if } 0<|z| \leq 1 / 4, \\ 1 & \text { if } z=0\end{cases}
$$

where $\sqrt{w}$ denotes the principal branch of the square root of $w$. In particular,

$$
a_{n}=\frac{1}{n+1}\left(\begin{array}{c}
2 n \\
n
\end{array}\right)=C_{n}
$$

the nth Catalan number.

Proof. Let $G$ be the function defined by the right-hand side of (3). Then $G$ is analytic in the disk $D=\{z:|z|<1 / 4\}, G$ is continuous on the closure $\bar{D}$ of $D$, and $G(0)=1$. Let $\left\{b_{n}\right\}$ be the sequence of Taylor coefficients of $G$ at the origin (i.e., the sequence of coefficients in the power series expansion of $G$ about 0 ). Then $b_{0}=1$ and, since $G(z)^{2}=(G(z)-1) / z$ when $0<|z|<1 / 4$,

$$
\sum_{n=0}^{\infty}\left(\sum_{k=0}^{n} b_{k} b_{n-k}\right) z^{n}=\sum_{n=0}^{\infty} b_{n} z^{n} \sum_{n=0}^{\infty} b_{n} z^{n}=\sum_{n=1}^{\infty} b_{n} z^{n-1}=\sum_{n=0}^{\infty} b_{n+1} z^{n} .
$$


It follows that

$$
b_{n+1}=\sum_{k=0}^{n} b_{k} b_{n-k} \quad(n=0,1,2, \ldots) .
$$

Thus, the sequences $\left\{a_{n}\right\}$ and $\left\{b_{n}\right\}$ satisfy the same recurrence relation and the same initial condition, from which we conclude that $a_{n}=b_{n}$ for all nonnegative integers $n$. In particular, $g(z)=G(z)$ for $z$ in $D$. Using the binomial series for $\sqrt{1-4 z}$, we see that

$$
\sqrt{1-4 z}=1-2 \sum_{n=1}^{\infty} \frac{1}{n}\left(\begin{array}{c}
2 n-2 \\
n-1
\end{array}\right) z^{n}
$$

whence

$$
g(z)=\sum_{n=1}^{\infty} \frac{1}{n}\left(\begin{array}{c}
2 n-2 \\
n-1
\end{array}\right) z^{n-1}=\sum_{n=0}^{\infty} C_{n} z^{n} .
$$

Stirling's formula asserts that $n ! \sim \sqrt{2 \pi} n^{n+1 / 2} e^{-n}[3]$. We infer that

$$
C_{n} \sim \pi^{-1 / 2} 4^{n} n^{-3 / 2}
$$

so the series on the right-hand side of (4) converges when $z=1 / 4$, which implies that it converges absolutely and uniformly on $\bar{D}$. Hence $g$ is also analytic in $D$ and continuous on $\bar{D}$. This ensures that $g$ and $G$ are just different representations of one and the same function on $\bar{D}$.

Theorem 3.2 and the discussion preceding it show that

$$
F(1,0)=(1-p) g(p(1-p)) .
$$

But $\sqrt{1-4 p(1-p)}=|2 p-1|$ when $0 \leq p \leq 1$. Hence

$$
F(1,0)= \begin{cases}1 & \text { if } 0 \leq p \leq 1 / 2, \\ \frac{1-p}{p} & \text { if } 1 / 2 \leq p \leq 1,\end{cases}
$$

(i.e., $F(1,0)=f(p)$, the function in (A)).

If we replace $p$ with an arbitrary complex number $z$, then the open set in which $F(1,0)$ is analytic is bounded by the curve with equation $|z(1-z)|=1 / 4$. This is the curve appearing in $(\mathrm{E})$.

We now see the connection between all that has been said and the functional relation in $(\mathrm{G})$. Observe that, by translation, $F(1,0)=F(2,1)$. Appealing to Proposition 2.2, we get

$$
\begin{aligned}
f(p) & =F(1,0)=(1-p)+p F(2,0)=(1-p)+p F(2,1) F(1,0) \\
& =(1-p)+p F(1,0)^{2}=(1-p)+p f(p)^{2},
\end{aligned}
$$

as asserted by $(\mathrm{G})$. (Of course, it is easy to check this relation directly from the definition of $f$.) 
By the way, notice that if $p>1 / 2$, then $F(1,0)<1$. In this event the random walk on $\mathbb{Z}_{+}^{0}$ is transient (i.e., the drunkard has some chance of avoiding the lake).

4. MORE GENERAL RANDOM WALKS ON $\mathbb{Z}_{+}^{\mathbf{0}}$. In this section and the next we extend the ideas developed earlier to more general random walks that yield connections similar to (A) through $(\mathrm{G})$ in the introduction. Consider the random walk on $\mathbb{Z}_{+}^{0}$ (analogous to $(\mathrm{F})$ ) with transition probabilities defined by $p(0,1)=1, p(1,2)=p$, $p(1,0)=1-p, p(n, n+1)=q$, and $p(n, n-1)=1-q$ for $n=2,3,4, \ldots$, where $p$ and $q$ are constants between 0 and 1 . The calculation of $F(2,1)$ never includes a path from 1 to 2 and is thus independent of $p$. Taking $p=q$ yields the same example as in section 3 , so that

$$
F(2,1)= \begin{cases}1 & \text { if } 0 \leq q \leq 1 / 2 \\ \frac{1-q}{q} & \text { if } 1 / 2 \leq q \leq 1\end{cases}
$$

For an arbitrary $p, F(1,0)=(1-p)+p F(2,1) F(1,0)$, and we obtain

$$
F(1,0)= \begin{cases}1 & \text { if } 0 \leq q \leq 1 / 2, \\ \frac{(1-p) q}{q-p+p q} & \text { if } 1 / 2 \leq q \leq 1 .\end{cases}
$$

We recall from Proposition 2.4 that the value of $F(1,0)$ determines the recurrence behavior of the corresponding random walk on $\mathbb{Z}_{+}^{0}$. Thus the random walk is transient if and only if $q>1 / 2$.

Let $k$ be any integer greater than 1 . In what follows, we focus on the case $q \geq 1 / 2$. Given such a $q$, we pick a specific value of $p$ : namely, we choose $p$ to be the unique solution in $(0,1)$ of the equation

$$
\frac{(1-p) q}{q-p+p q}=\sqrt[k-1]{\frac{1-q}{q}}
$$

Then $p$ belongs to $[1 / k, 1)$. In fact, this establishes a one-to-one correspondence $q \mapsto p$ between $(1 / 2,1)$ and $(1 / k, 1)$, as we'll see later.

The reason for choosing $p$ to satisfy (5) is to get the identity

$$
F(1,0)=1-p+p F(1,0)^{k} .
$$

Let $f(p)=F(1,0)$, where $q$ in $(1 / 2,1)$ and $p$ in $(1 / k, 1)$ are related by (5). Then (6) translates to the generalization of the functional equation $(\mathrm{G})$ :

$$
f(p)=1-p+p f(p)^{k} .
$$

Observe that $f(p)=1$ satisfies (7) and that if $f(p)$ satisfies (7) and $f(p) \neq 1$, then

$$
f(p)^{k-1}+\cdots+f(p)+1=\frac{1-f(p)^{k}}{1-f(p)}=\frac{1}{p} .
$$

If, furthermore, $0 \leq p \leq 1 / k$ and $f(p) \neq 1$, then (8) implies that $f(p)>1$, which contradicts the fact that $f(p)$ is a probability. Thus, $f(p)=1$ when $0 \leq p \leq 1 / k$. On the other hand, we will show presently that $q>1 / 2$ if $p>1 / k$, whence $F(1,0)<1$ 
by Proposition 2.4. Therefore $f(p)=y$, where $y$ in $[0,1]$ satisfies $y^{k-1}+\cdots+$ $y+1=1 / p$. That is, we get the analogue of (A) for the general case $k(\geq 2)$ :

$$
f(p)= \begin{cases}1 & \text { if } p \in[0,1 / k] \\ y & \text { if } p \in[1 / k, 1]\end{cases}
$$

where $y$ is the unique positive solution to $\sum_{j=0}^{k-1} y^{j}=1 / p$. In the case $k=2$ we recover (1) and in the case $k=3$ (see Figure 3), we have

$$
f(p)= \begin{cases}1 & \text { if } p \in[0,1 / 3], \\ \frac{\sqrt{\frac{4}{p}-3}-1}{2} & \text { if } p \in[1 / 3,1] .\end{cases}
$$

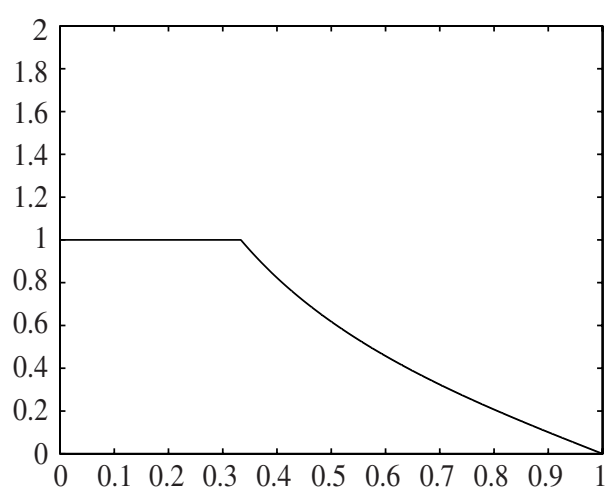

Figure 3.

Now consider $F(1,0)$ as a function of $q$ in $(1 / 2,1)$. Then by (5) we have

$$
F(2,1)=\frac{1-q}{q}=\left(\frac{(1-p) q}{q-p+p q}\right)^{k-1}=F(1,0)^{k-1},
$$

so $F(1,0)$ is a decreasing function of $q$. Writing

$$
s=\sqrt[k-1]{\frac{1-q}{q}}
$$

and solving for $p$ in (5) yields

$$
p=\frac{q(1-s)}{q(1+s)-s} .
$$

For $q=1, s=0$ and $p=1$. For $q$ approaching $1 / 2$ from the right, $s$ approaches 1 from the right, so an application of L'Hôpital's rule shows that $p$ approaches $1 / k$. Thus (5) gives a one-to-one correspondence between $p$ in $(1 / k, 1)$ and $q$ in $(1 / 2,1)$.

For a fixed positive integer $k$ the numbers $a_{n, k}$ given by

$$
a_{n, k}=\frac{1}{(k-1) n+1}\left(\begin{array}{c}
k n \\
n
\end{array}\right) \quad(n=0,1,2, \ldots)
$$


are known as the generalized Catalan numbers (with parameter $k$ ). For $k=2$ they are precisely the Catalan numbers.

The Lagrange-Bürmann inversion formula considers $\zeta=w+\alpha \varphi(\zeta)$ for a smooth function $\varphi$ and states that

$$
\zeta=w+\sum_{n=1}^{\infty} \frac{\alpha^{n}}{n !} \frac{d^{n-1}}{d w^{n-1}}\left[\varphi(w)^{n}\right]
$$

for $\alpha$ sufficiently small, where by

$$
\frac{d^{n-1}}{d w^{n-1}} \varphi(w)^{n}
$$

we mean

$$
\left.\frac{d^{n-1}}{d \zeta^{n-1}} \varphi(\zeta)^{n}\right|_{\zeta=w}
$$

In particular, for $\varphi(\zeta)=\zeta^{k}$ (i.e., for $\zeta=w+\alpha \zeta^{k}$ ), (10) yields

$$
\begin{aligned}
\zeta & =w+\sum_{n=1}^{\infty} \frac{\alpha^{n}}{n !} \frac{d^{n-1}}{d w^{n-1}} w^{k n} \\
& =\sum_{n=0}^{\infty} \frac{1}{(k-1) n+1}\left(\begin{array}{c}
k n \\
n
\end{array}\right) \alpha^{n} w^{(k-1) n+1} \\
& =\sum_{n=0}^{\infty} a_{n, k} \alpha^{n} w^{(k-1) n+1} .
\end{aligned}
$$

This inversion formula appears in many books and articles, including [6, prob. 211], [7, p. 175], [5, p. 406], and [4, p. 72]. The Lagrange-Bürmann inversion formula applied to $\zeta=f(p), w=1-p$, and $\alpha=p$, yields an analogue of $(\mathrm{B})$ :

$$
f(p)=(1-p) \sum_{n=0}^{\infty} a_{n, k}\left[p(1-p)^{k-1}\right]^{n} .
$$

Let us consider the series that arises from (11) when $p$ is replaced with a complex variable $z$. By Stirling's formula,

$$
a_{n, k} \sim \sqrt{\frac{k}{2 \pi n^{3}(k-1)^{3}}}\left(\frac{k^{k}}{(k-1)^{k-1}}\right)^{n},
$$

so

$$
\sqrt[n]{a_{n, k}} \sim \frac{k^{k}}{(k-1)^{k-1}} .
$$

By the root test and Stirling's formula, the set of convergence of the series in question is the set $R$ described by

$$
R=\left\{z:\left|z(1-z)^{k-1}\right| \leq(k-1)^{k-1} / k^{k}\right\},
$$


whose boundary $\Gamma$ looks like a figure-eight that is symmetric with respect to the real axis. We will show that, in fact, the complement of $\Gamma$ has three connected components, two bounded components $D_{0}$ and $D_{1}$ and one unbounded component $D_{2}$. The set $R$ is the closure of the union of $D_{0}$ and $D_{1}$. On the component $D_{0}$ containing 0 the sum of the series is identically 1 , while on $D_{1}$ the series sums to a nonconstant analytic function of $z$ determined by the equation $w=1-z+z w^{k}$. (This follows from (9) and the fact that the sum of the series is analytic in both $D_{0}$ and $D_{1}$.) When $z$ lies in $D_{2}$, the series is divergent. (For the case $k=3$, see Figure 4. In this instance the sum $f$ of the series in $D_{1}$ is given by

$$
f(z)=\frac{\sqrt{4 z^{-1}-3}-1}{2},
$$

where the radical signifies the principal square root.) This is the analogue of (E).

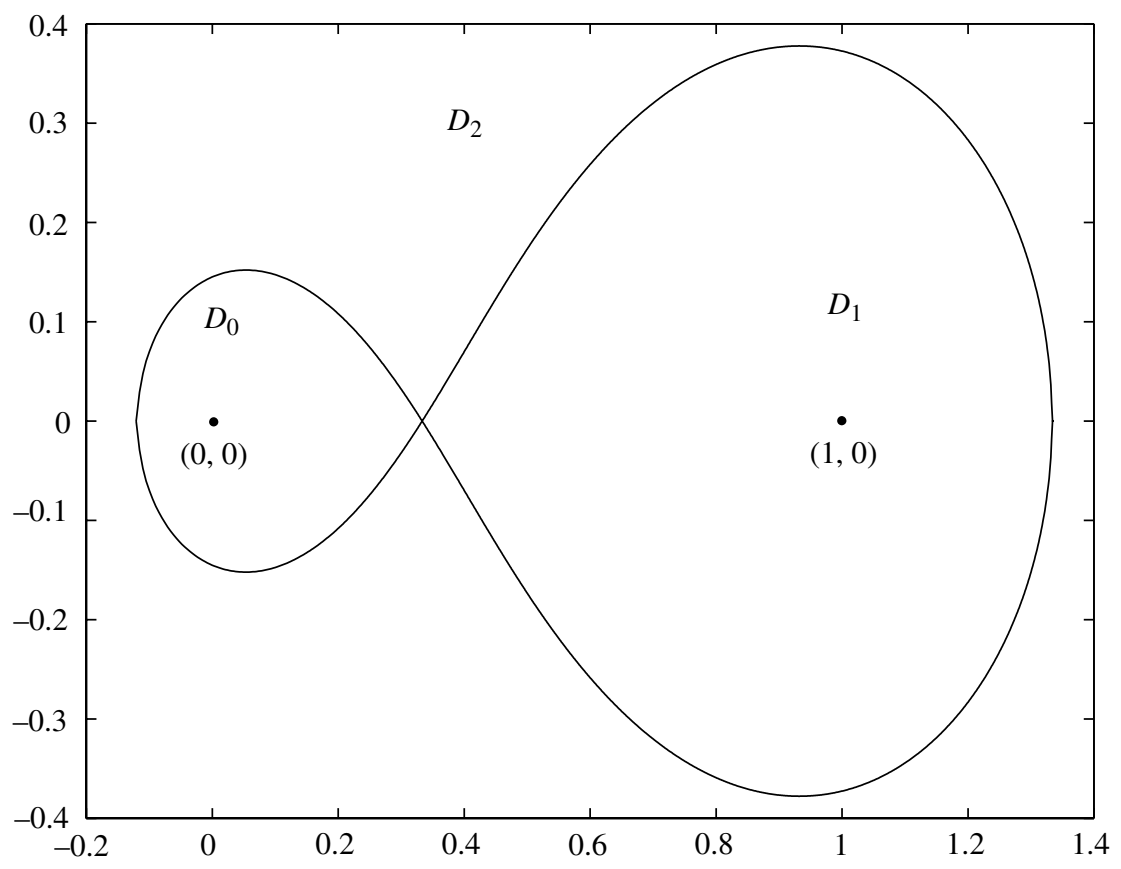

Figure 4.

The curve $\Gamma$ is described by the equation $h(x, y)=0$, with

$$
h(x, y)=\left(x^{2}+y^{2}\right)\left((1-x)^{2}+y^{2}\right)^{k-1}-\frac{(k-1)^{2(k-1)}}{k^{2 k}} .
$$

Thus $\Gamma$ crosses the $x$-axis if and only if $g(x)=x(1-x)^{k-1}= \pm a$, where

$$
a=\frac{1}{k}\left(1-\frac{1}{k}\right)^{k-1}
$$

Observe that on $(-\infty, 0]$ the function $g$ decreases to $-\infty$ as $x \rightarrow-\infty$, on $[0,1 / k]$ it increases from 0 to $a$, and on $[1 / k, 1]$ it decreases from $a$ to 0 . On the other hand, 
on $[1, \infty)$ the function $(-1)^{k-1} g$ increases to $\infty$. Thus $g(x)= \pm a$ for exactly three values $x_{1}, x_{2}$, and $x_{3}$ of $x$, with $x_{1}<0<x_{2}=1 / k<1<x_{3}$.

Fixing any value of $x$, consider the function $y \mapsto h(x, y)$. Elementary calculus shows that this function is decreasing on $(-\infty, 0)$ and increasing on $(0, \infty)$. Thus, if $x<x_{1}$ or $x>x_{3}, h(x, y)>0$. If $x$ is any of the numbers $x_{1}, x_{2}$, or $x_{3}$, then $h(x, y)=0$ only at $y=0$. Finally, consider the case of $x$ with $x_{1}<x<x_{2}$ or $x_{2}<x<x_{3}$. As we have seen, $h(x, 0)<0$. Furthermore, $h(x, y) \rightarrow+\infty$ as $|y| \rightarrow \infty$. It follows that there are exactly two values of $y$ such that $h(x, y)=0$. The upshot of these considerations: $\Gamma$ indeed looks like a figure-eight, with its left extreme at $\left(x_{1}, 0\right)$, a double point at $\left(x_{2}, 0\right)$, and its right extreme at $\left(x_{3}, 0\right)$.

Observe that when $y>0$ and $y \neq 1$ the sign of $\sum_{j=0}^{k-1} y^{j}$ is always positive. Thus, the equation

$$
\sum_{j=0}^{k-1} y^{j}=\frac{1}{x}
$$

cannot have negative solutions $x$. But the component $D_{0}$ contains negative values of $x$. Accordingly, on $D_{0}$ the value of $y=F(1,0)$ must be identically 1 .

\section{GENERALIZED CATALAN NUMBERS AND THEIR PROBABILISTIC} INTERPRETATION. The generalized Catalan numbers have numerous combinatorial interpretations (see [5] and [4]). Some of the formulas that we derive here (including the recurrence relation in Proposition 5.1) can be found in the elegant paper [4]. The following is the generalization of (D):

Proposition 5.1. The generalized Catalan numbers $a_{n, k}$ satisfy the recurrence relation

$$
a_{n+1, k}=\sum_{i_{1}+\cdots+i_{k}=n} a_{i_{1}, k} \ldots a_{i_{k}, k} \quad(n \geq 0),
$$

where $a_{0, k}=1$.

Proof. Fix a positive integer $k$, and let $b_{n}$ be the sequence defined by the recurrence relation (12) with $b_{0}=1$. If

$$
G(w)=\sum_{n=0}^{\infty} b_{n} w^{n}
$$

then $G$ is analytic in the disk $D=\left\{w:|w|<k^{k} /(k-1)^{k-1}\right\}$ and

$$
\begin{aligned}
G(w)^{k} & =\sum_{n=0}^{\infty} \sum_{i_{1}+\cdots+i_{k}=n} b_{i_{1}} \ldots b_{i_{k}} w^{n} \\
& =\sum_{n=0}^{\infty} b_{n+1} w^{n}=\frac{1}{w}\left(\sum_{n=0}^{\infty} b_{n} w^{n}-1\right) \\
& =\frac{1}{w}(G(w)-1) .
\end{aligned}
$$


For $z$ in the interior of set $R, w=z(1-z)^{k-1}$ belongs to the disk $D$, and $F(z)=$ $(1-z) G(w)$ yields $F(z)=1-z+z F(z)^{k}$. Using (11), we obtain

$$
G(w)=\sum_{n=0}^{\infty} a_{n, k} w^{n} .
$$

Hence $b_{n}=a_{n, k}$ for all nonnegative $n$, establishing the result.

We next consider the cautious drunkard's walk on $\mathbb{Z}_{+}^{0}$ with parameter a fixed integer $k$ greater than 1: at each stage the drunkard can move exactly $k-1$ steps forward or move one step backward. This drunkard is more cautious, because at each stage he may move several steps away from the lake, but just one toward it. We will see that $a_{m, k}$ is exactly the number of such paths from 1 to 1 with $\mathrm{km}$ moves (i.e., the number of paths $\left[c_{0}, \ldots, c_{k m}\right]$ in $\mathbb{Z}_{+}$with $c_{0}=c_{k m}=1$,

$$
c_{j+1}=c_{j}+\frac{k-2}{2} \pm \frac{k}{2}
$$

and $c_{j}>0$ for each $j$.)

Here is the proof in the special case $k=3$. A path $c$ of length $n$ is now a sequence $\left[c_{0}, c_{1}, \ldots, c_{n}\right]$, where $c_{i+1}=c_{i}+2$ or $c_{i+1}=c_{i}-1$. If $c$ is a loop (i.e., if $c_{0}=c_{n}$ ), then $n$ is a multiple of three: when a path begins and ends at the same point and has $s$ forward moves, it must have $2 s$ backward moves, hence $3 s$ moves altogether. If $c_{n}=c_{0}+1$, then $\left[c_{0}, c_{1}, \ldots, c_{n}, c_{0}\right]$ is a loop, so 3 must divide $n+1$, whereas if $c_{n}=c_{0}+2$, then $\left[c_{0}, c_{1}, \ldots, c_{n}, c_{0}+1, c_{0}\right]$ is a loop, in which event 3 divides $n+2$.

Let $A_{m}^{j}$ be the set of all paths in $\mathbb{Z}_{+}$of length $3 m$ that begin and end at $j$ and do not contain $j-1$, and let $a_{m}$ be the cardinality of $A_{m}^{j}$. Fix $c=\left[c_{0}, \ldots, c_{3 m}\right]$ in $A_{m}^{1}$. Let $c_{3 m_{1}}$ be the first occurrence of 1 in $c$, so $m_{1} \leq m$. Let $c_{3 m_{2}-1}$ be the first occurrence of 2 in $c$. Since $c_{3 m_{1}-1}=2$, it follows that $m_{2} \leq m_{1}$. Note that $c_{1}=3$ and $c_{3 m_{2}-2}=3$. Thus $c$ is the concatenation of $1, \gamma_{3}, \gamma_{2}$, and $\gamma_{1}$, where $\gamma_{3}=\left[c_{1}, \ldots, c_{3 m_{2}-2}\right]$ belongs to $A_{m_{2}-1}^{3}, \gamma_{2}=\left[c_{3 m_{2}-1}, \ldots, c_{3 m_{1}-1}\right]$ to $A_{m_{1}-m_{2}}^{2}$, and $\gamma_{1}=\left[c_{3 m_{1}}, \ldots, c_{3 m}\right]$ to $A_{m-m_{1}}^{1}$. This furnishes a unique representation of any loop $c$ in $A_{m}^{1}$. On the other hand, any choice of $\gamma_{3}$ from $A_{n_{3}}^{3}, \gamma_{2}$ from $A_{n_{2}}^{2}$, and $\gamma_{1}$ from $A_{n_{1}}^{1}$, where $n_{1}+n_{2}+n_{3}=m-1$, yields a unique path $c=1 \cdot \gamma_{3} \cdot \gamma_{2} \cdot \gamma_{1}$ in $A_{m}^{1}$. Thus

$$
a_{n+1}=\sum_{n_{1}+n_{2}+n_{3}=n} a_{n_{1}} a_{n_{2}} a_{n_{3}} .
$$

Since $a_{0}=1$, by invoking Proposition 5.1 we deduce that $a_{n}$ is the generalized Catalan number $a_{n, 3}$.

As a consequence of the interpretation of $a_{n, k}$ as the number of "cautious" paths in $\mathbb{Z}_{+}$of length $k n$ starting and ending at 1 , the generalized Catalan numbers $a_{n, k}$ are seen to solve the following ballot problem: In an election in which candidate A gets $n$ votes and candidate $B$ gets $(k-1) n$ votes, what is the number of ways of counting the ballots so that candidate $B$ never has more than $k-1$ times as many votes as candidate A?

As in the case of the Catalan numbers, the generalized Catalan numbers can be derived by means of a Pascal-like triangle as follows. Fix an integer $k$ bigger than 1 . For $n$ in $\mathbb{Z}_{+}^{0}$ and $m$ in $\mathbb{Z}_{+}$, let $\gamma_{n, m}$ be the number of cautious drunkard paths (with parameter $k$ ) in $\mathbb{Z}_{+}$of length $n$ that begin at $m$ and end at 1 . Since any path that begins at $m$ must move to $m-1$ or to $m+k-1$ in the next step, $\gamma_{n, m}$ must satisfy the 
recurrence relation

$$
\gamma_{n, m}=\gamma_{n-1, m-1}+\gamma_{n-1, m+k-1} .
$$

Furthermore, $\gamma_{n, n+1}=1$ for any positive integer $n$. Observe that the generalized Catalan numbers are given by $\gamma_{k n, 1}$. Now form the one-sided Pascal triangle by considering the values of $\gamma_{n, m}$. In each row, add the entry on the upper left side to the entry $k-1$ steps to the right. The generalized Catalan numbers are the nonzero entries in the first column. We display the Pascal triangle for the case $k=3$, where the corresponding sequence of generalized Catalan numbers is $1,1,3,12,55,273, \ldots$ :

$\begin{array}{cccccccccc}1 & & & & & & & & & \\ 0 & 1 & & & & & & & & \\ 0 & 0 & 1 & & & & & & & \\ 1 & 0 & 0 & 1 & & & & & & \\ 0 & 2 & 0 & 0 & 1 & & & & & \\ 0 & 0 & 3 & 0 & 0 & 1 & & & & \\ 3 & 0 & 0 & 4 & 0 & 0 & 1 & & & \\ 0 & 7 & 0 & 0 & 5 & 0 & 0 & 1 & & \\ 0 & 0 & 12 & 0 & 0 & 6 & 0 & 0 & 1 & \\ 12 & 0 & 0 & 18 & 0 & 0 & 7 & 0 & 0 & 1 \\ \ldots & \ldots & \ldots & \ldots & \ldots & \ldots & \ldots & \ldots & \ldots & \ldots\end{array}$

This is the generalization of $(\mathrm{C})$.

We next give a probabilistic interpretation of the generalized Catalan numbers corresponding to the case $k=3$, which turns out to be equivalent to the description of the cautious drunkard's walk outlined earlier in the section. Specifically, we are going to present a simple infinite directed graph on a horizontal strip whose edges are horizontal, vertical, or diagonal segments and whose edge-probabilities are chosen so that the number of paths of length $n$ that start and end at the same vertex and never move to a vertex to the left of the starting vertex is the generalized Catalan number $a_{n, 3}$. A similar construction can be carried out to yield a graph corresponding to any generalized Catalan number $a_{n, k}$.

Consider the directed graph $G$ in Figure 5. Label the vertices on the graph by means of the even (respectively, odd) integers on the bottom line (respectively, top line). Assume that the rightward probabilities along the horizontal direction are all equal to some fixed number $p$ in $(0,1)$ and that the probability assigned to each downward-oriented edge and to each upward-directed diagonal edge is $1-p$. The nearest-neighbor transition probabilities are 0 in the opposite of the indicated directions.

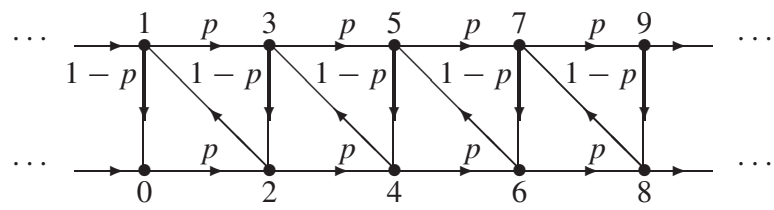

Figure 5. Graph associated with the generalized Catalan numbers $a_{n, 3}$.

We wish to count the number of directed paths on $G$ of fixed length $n$ that never move to a vertex with a smaller label than the one of the starting vertex. Observe 
that such a path has the form $c=\left[c_{0}, c_{1}, \ldots, c_{n}\right]$, where $c_{i+1}=c_{i}+2$ or $c_{i}-1$ for $i=0, \ldots, n-1$, and $c_{i} \geq c_{0}$ for all $i$. If we assume that $c_{0}$ lies in $\mathbb{Z}_{+}$, then $c$ represents a path for the cautious drunkard's walk. Conversely, any path for the cautious drunkard's walk yields such a path in $G$. Thus the number of directed cycles in $G$ that never move to a vertex with a smaller label than that of the starting vertex is $a_{n, 3}$. Let $r$ be the probability that a path starting at 1 visits 0 . It follows from (9) and (11) that $r=1$ if and only if $p<1 / k$.

We close this section with an observation about Catalan numbers. It applies whether they be the classical ones or their generalized versions.

The fact that the function

$$
f(z)=(1-z) \sum_{n=0}^{\infty} a_{n, k}\left[z(1-z)^{k-1}\right]^{n}
$$

is identically 1 near 0 means that, after suitable expansion and rearrangement of its terms, the series

$$
\sum_{n=0}^{\infty} a_{n, k}\left[z(1-z)^{k-1}\right]^{n}
$$

reduces to the geometric series $\sum_{m=0}^{\infty} z^{m}$ for $z$ sufficiently close to zero. Initially we assumed that this was a very simple observation. Instead we found that it leads to an unexpected relation among the generalized Catalan numbers that does not seem to have an obvious combinatorial proof.

Theorem 5.1. For all integers $k \geq 2$ and $m \geq 0$

$$
\sum_{n=\lceil m / k\rceil}^{m}(-1)^{n} a_{n, k}\left(\begin{array}{c}
k n-n \\
k n-m
\end{array}\right)=(-1)^{m},
$$

where $\lceil x\rceil$ signifies the smallest integer greater than or equal to $x$.

This relation holds trivially for $k=1$. Even in the case of the classical Catalan numbers, the relation

$$
\sum_{n=\lceil m / 2\rceil}^{m}(-1)^{n} C_{n}\left(\begin{array}{c}
n \\
2 n-m
\end{array}\right)=(-1)^{m}
$$

does not seem to have a simple proof. We have not found this relation in the literature, but we certainly do not preclude the possibility that it is both in the literature and has a simple proof that has eluded us.

Proof. Expanding the terms $z^{n}(1-z)^{(k-1) n}$ we rewrite (13) as

$$
\sum_{n=0}^{\infty} a_{n, k} \sum_{t=0}^{n(k-1)}\left(\begin{array}{c}
(k-1) n \\
t
\end{array}\right)(-1)^{n(k-1)-t} z^{n k-t},
$$

which (since the series is absolutely convergent in a neighborhood of 0 ) we can rewrite in the form 


$$
\sum_{m=0}^{\infty}(-1)^{m}\left[\sum_{n=\lceil m / k\rceil}^{m}(-1)^{n} a_{n, k}\left(\begin{array}{c}
k n-n \\
k n-m
\end{array}\right)\right] z^{m} .
$$

Because this series coincides with the geometric series in a neighborhood of the origin, we arrive at the stated result.

In particular, we obtain a new simple recursive definition of the generalized Catalan numbers:

$$
a_{m, k}=1-(-1)^{m} \sum_{n=\lceil m / k\rceil}^{m-1}(-1)^{n} a_{n, k}\left(\begin{array}{c}
k n-n \\
k n-m
\end{array}\right)
$$

for $m=1,2, \ldots$

6. THE EXAMPLE THAT INSPIRED THIS PAPER. The function $H^{*}$ in Theorem 2.1 says a great deal about whether or not a random walk on a tree is transient, but it does not quite characterize transience. In this section we give an example that makes this limitation of $H^{*}$ precise. The example was presented originally in [1, Example 5.1] without proof. We now describe a one-parameter family of random walks on a homogeneous tree of degree three. In each of them the inward probabilities remain the same-so $H^{*}$ is identical in all cases_-but the random walks can be transient or recurrent depending upon the parameter.

Recall the recurrent random walk on a homogeneous tree of degree three described in Example 2.2. We will modify the transition probabilities along a single ray that, for simplicity, we identify with $\mathbb{Z}_{+}^{0}$.

Consider the tree $T_{3}$ of Example 2.2 with root $e=0$, and the ray

$$
\rho=[0=e, 1,2, \ldots] .
$$

Fix $p$ in $(0,2 / 3)$. Let $T$ be the same tree as $T_{3}$, but with the transition probabilities $p(v, u)$ modified as follows when $v=n$ with $n \geq 1$ : let $p(n, n-1)=1 / 3$, $p(n, n+1)=p$, and $p\left(v_{n}, w_{n}\right)=q=2 / 3-p$, where $w_{n}$ is the neighbor of $n$ different from $n \pm 1$ (see Figures 6 and 7).

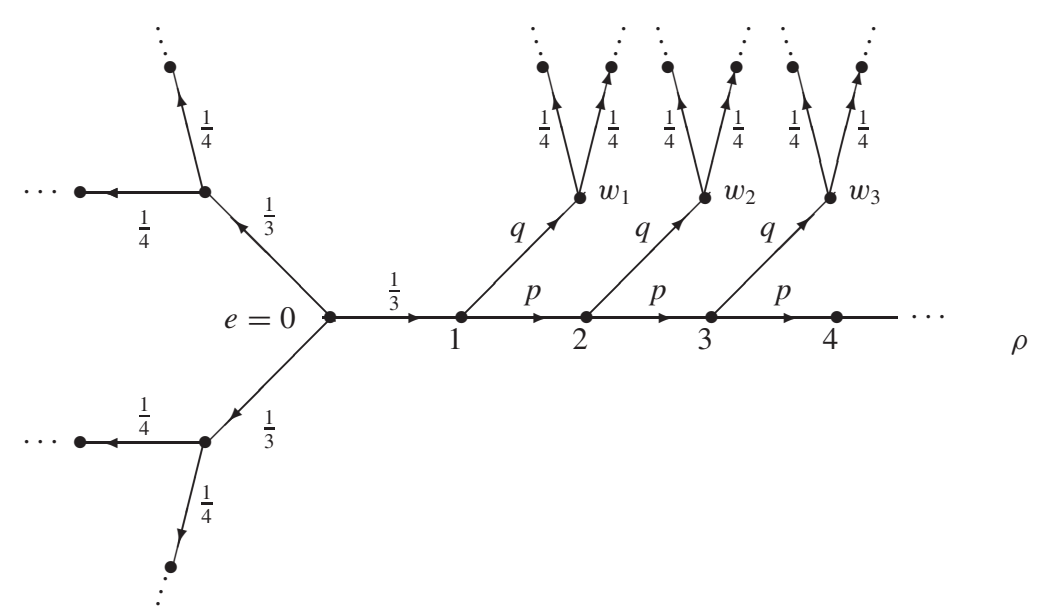

Figure 6. Outward probabilities on $T$. 


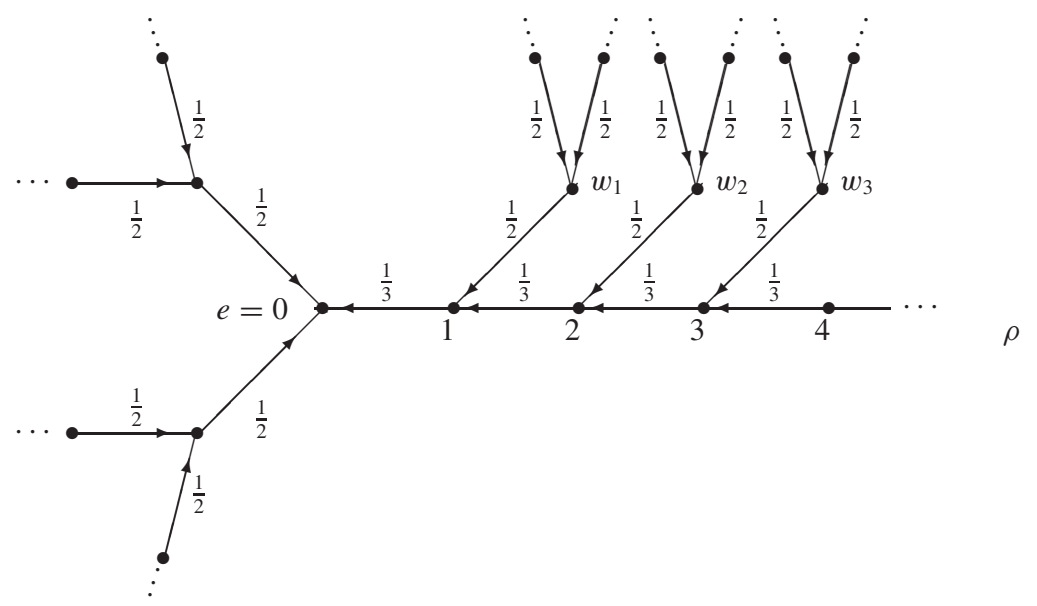

Figure 7. Inward probabilities on $T$.

Notice that for each vertex $v$ of $T$ that does not lie on $\rho$ the probabilities on the set $\Gamma_{v, v^{-}}$(see section 2) with respect to $T$ are the same as those on the corresponding set with respect to $T_{3}$. Therefore $F\left(v, v^{-}\right)$is the same for both trees. Since the random walk on $T_{3}$ is recurrent, $F\left(v, v^{-}\right)=1$. In particular, $F\left(w_{n}, n\right)=1$ for all $n$ in $\mathbb{N}$.

By Proposition 2.2 we have (for the function $F$ associated with $T$ )

$$
F(1,0)=1 / 3+p F(2,1) F(1,0)+q F\left(w_{1}, 1\right) F(1,0),
$$

yielding $F(1,0)=1 / 3+p F(1,0)^{2}+q F(1,0)$. Since $q=2 / 3-p$, we obtain

$$
F(1,0)=\frac{1}{1+3 p}+\frac{3 p}{1+3 p} F(1,0)^{2} .
$$

The Lagrange-Bürmann inversion formula tells us that

$$
\begin{aligned}
F(1,0) & =\sum_{n=0}^{\infty} C_{n}\left(\frac{3 p}{1+3 p}\right)^{n}\left(\frac{1}{1+3 p}\right)^{n+1} \\
& =\frac{1}{1+3 p} \sum_{n=0}^{\infty} C_{n}\left[\frac{3 p}{(1+3 p)^{2}}\right]^{n}
\end{aligned}
$$

where the coefficients $C_{n}$ are the Catalan numbers. Therefore formally it would appear that

$$
F(1,0)=f\left(\frac{3 p}{1+3 p}\right)= \begin{cases}1 & \text { if } 0<p \leq \frac{1}{3} \\ 1 /(3 p) & \text { if } \frac{1}{3} \leq p \leq \frac{2}{3}\end{cases}
$$

where $f$ is the function in (1). The problem with this argument is that the LagrangeBürmann inversion formula is valid only in some neighborhood of 0 , not necessarily on all of $[0,2 / 3]$. The result in (14), however, is correct for all $p$ in $[0,2 / 3]$, as the following probabilistic argument shows.

We calculate $F(1,0)$ by studying all paths in $T$ from 1 to 0 that do not visit 0 before the last step. Each such path $c$ has a unique representation

$$
c=c_{0} \cdot \gamma_{0} \cdot c_{1} \cdot \gamma_{1} \cdots c_{2 n} \cdot \gamma_{2 n} \cdot 0
$$


where $c_{0}=c_{2 n}=1,\left|c_{i}-c_{i-1}\right|=1$, and $\gamma_{i}$ is a path from $c_{i}$ to itself that does not pass through any other integer $j$. Fixing $n$, let $\Gamma^{n}$ denote the set of all such paths. In light of the restrictions on the $c_{i}$, we see that there are exactly $C_{n}$ choices for the ordered set $\left(c_{0}, c_{1}, \ldots, c_{2 n}, 0\right)$, each carrying exactly the same probability. This probability is the product of several factors: there are $n$ times when $c_{i+1}>c_{i}$, contributing $p^{n}$; there are $n+1$ times when $c_{i+1}<c_{i}$, contributing $\left(\frac{1}{3}\right)^{n+1}$; and finally the set of all possible $\gamma_{i}$ contributes 1 (the empty path) plus $\left(q F\left(w_{c_{i}}, c_{i}\right)\right)^{k}$ (for a path going from $c_{i}$ to $w_{c_{i}}$ and eventually back to $c_{i}$, having touched $c_{i}$ exactly $k$ times) for each $k$. Recalling that $F\left(w_{c_{i}}, c_{i}\right)=1$, we get

$$
p^{n}\left(\frac{1}{3}\right)^{n+1}\left(\sum_{k=0}^{\infty} q^{k}\right)^{2 n+1}=\frac{p^{n}}{3^{n+1}} \frac{1}{(1-q)^{2 n+1}}
$$

as the sum of the path probabilities for fixed $c_{0}, \ldots, c_{n+1}$. We calculate this sum to be

$$
\frac{1}{3} \frac{1}{1-q}\left[\frac{p}{3(1-q)^{2}}\right]^{n}=\frac{1}{1+3 p}\left[\frac{3 p}{(1+3 p)^{2}}\right]^{n},
$$

since $3(1-q)=1+3 p$. Thus the sum of the path probabilities over $\Gamma^{n}$ is

$$
C_{n} \frac{1}{1+3 p}\left[\frac{3 p}{(1+3 p)^{2}}\right]^{n},
$$

which establishes (14). Consequently, the random walk on $T$ is recurrent when $0<p \leq 1 / 3$ and transient when $1 / 3<p \leq 2 / 3$.

Since $p\left(v, v^{-}\right)=1 / 2$ whenever $v$ is not a vertex on $\rho$, if $\rho^{\prime}$ is any ray in $T$ other than $\rho$, then $\epsilon_{n}\left(\rho^{\prime}\right)=1$ for all $n$ sufficiently large. Accordingly, $H^{*}\left(\rho^{\prime}\right)=\infty$. Yet $\epsilon_{n}(\rho)=1 / 2$ for every positive integer $n$, so

$$
H^{*}(\rho)=\sum_{k=0}^{\infty}\left(\frac{1}{2}\right)^{k}=2<\infty
$$

regardless of the value of $p$, whence $H^{*}$ does not itself determine whether or not the random walk is transient.

Incidentally, $p=2 / 3$ means that $q=0$, disconnecting the tree. In this case, $F(1,0)$ is calculated on a tree that is $\mathbb{Z}_{+}^{0}$ with transition probabilities $p(n, n+1)=2 / 3$ and $p(n, n-1)=1 / 3$, giving $F(1,0)=1 / 2$. Finally, letting $t=3 p$, we obtain

$$
F(1,0)=\frac{1}{1+t} \sum_{n=0}^{\infty} C_{n}\left[\frac{t}{(1+t)^{2}}\right]^{n}
$$

defined when $t /(1+t)^{2} \leq 1 / 4$.

We were originally drawn to this problem by the strangeness of the fact that the series

$$
\frac{1}{1+t} \sum_{n=0}^{\infty} C_{n}\left[\frac{t}{(1+t)^{2}}\right]^{n}
$$

converges on $[0, \infty)$ but takes the value 1 when $0 \leq t \leq 1$ and $1 / t$ when $t \geq 1$. The mysterious change in behavior becomes clear by looking at this as a series in a complex 
variable. We know that

$$
\frac{1}{1+z} \sum_{n=0}^{\infty} C_{n}\left[\frac{z}{(1+z)^{2}}\right]^{n}
$$

converges to a function $f(z)$ exactly when $\left|z /(1+z)^{2}\right| \leq 1 / 4$. The complement of the curve

$$
\Gamma=\left\{z:\left|\frac{z}{(1+z)^{2}}\right|=\frac{1}{4}\right\}
$$

is the disjoint union of domains $D_{0}, D_{1}$, and $D_{2}$, where $D_{2}$ is unbounded, $D_{0}$ is the bounded connected component containing 0 , and $D_{1}$ is the other bounded component (see Figure 8).

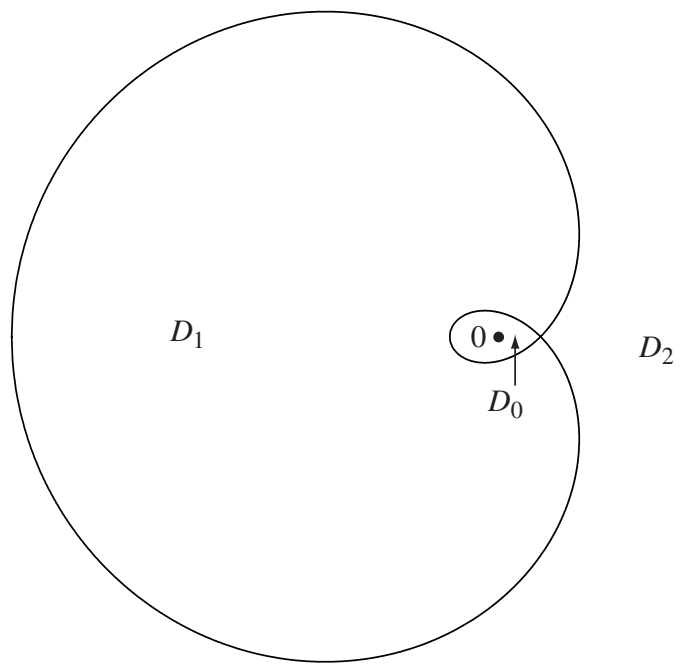

Figure 8.

The real line intersects $\Gamma$ at the points $-3-2 \sqrt{2},-3+2 \sqrt{2}$, and 1 . The function $f$ is defined and bounded by 1 on the complement of $D_{1}$. On $D_{0}$ it is identically 1 and $f(z)=1 / z$ for $z$ in $D_{2}$. In particular, $f$ is continuous on $[0, \infty)$ and analytic on $[0,1) \cup(1, \infty)$, but it is not analytic at 1 .

The surprising conclusion is that this example mathematically reduces to the study of the drunkard's walk on $\mathbb{Z}_{+}^{0}$.

ACKNOWLEDGMENTS. We wish to express our deepest gratitude to the referee for his insightful suggestions on how to improve our manuscript. We also thank Glenn Easley for his help with the graphics.

\section{REFERENCES}

1. I. Bajunaid, J. M. Cohen, F. Colonna, and D. Singman, Trees as Brelot spaces, Adv. Appl. Math. 30 (2003) 706-745.

2. P. Cartier, Fonctions harmoniques sur un arbre, Sympos. Math. 9 (1972) 203-270.

3. W. Feller, An Introduction to Probability Theory and Its Applications, vol. 1, 3rd ed., John Wiley \& Sons, New York, 1968. 
4. P. Hilton and J. Pederson, Catalan numbers, their generalization, and their uses, Math. Int. 13 (1991) 64-75.

5. D. A. Klarner, Correspondences between plane trees and binary sequences, J. Comb. Theory 9 (1970) 401-411.

6. G. Pólya, and G. Szegö, Problems and Theorems in Analysis I, Springer-Verlag, New York, 1976.

7. R. P. Stanley, Enumerative Combinatorics, vol. 2, Cambridge University Press, Cambridge, 1999.

8. E. W. Weisstein, CRC Concise Encyclopedia of Mathematics, Chapman \& Hall/CRC, Boca Raton, FL, 1999.

IBTESAM BAJUNAID is an assistant professor of mathematics at King Saud University in Saudi Arabia. She received her Ph.D. from King Saud University in 1999 under the direction of Victor Anandam. She was the first woman to be granted a Ph.D. in mathematics from King Saud University. Her primary mathematical interest is potential theory, and she is currently collaborating with Dr. Anandam on polypotentials on trees.

King Saud University, Riyadh, Saudi Arabia

ibajunaid@hotmail.com

JOEL COHEN has been professor of mathematics at the University of Maryland since 1978. He received his Ph.D. in mathematics from MIT in 1966 and also taught at the University of Chicago and the University of Pennsylvania. His early work in algebraic topology and low-dimensional complexes led to an interest in combinatorial group theory. The study of free groups led naturally to trees. The trees then led him astray to functional analysis, harmonic analysis, integral geometry, and potential theory. In his spare time he is active in politics, and is national chair of the liberal political group Americans for Democratic Action.

Department of Mathematics, The University of Maryland, College Park, MD 20742

jcohen@umd.edu

FLAVIA COLONNA is professor of mathematics at George Mason University. Before her tenure there, she was a junior faculty member at the University of Bari, Italy. She received a Ph.D. in mathematics from the University of Maryland in 1985. Her research interests include complex and harmonic analysis, potential theory, integral geometry, and image processing. She has been involved in various outreach programs for girls in sixth through eighth grades and serves as a judge in the Mathcounts National Competition.

Department of Mathematics, George Mason University, Fairfax, VA 22030

fcolonna@gmu.edu

DAVID SINGMAN is professor of mathematics at George Mason University. He received a Ph.D. degree in mathematics from McGill University in 1980. His research interests include potential theory in classical, axiomatic, and discrete settings.

Department of Mathematics, George Mason University, Fairfax, VA 22030 dsingman@gmu.edu 